Nicolas D'ANDRÈs, Socrate néoplatonicien. Une science de l'amour dans le Commentaire de Proclus sur le Premier Alcibiade

Paris, Vrin, 2020 (Textes et traditions), 304 p., ISBN : 978-2-7116-2955-8

\title{
Corentin Tresnie
}

\section{(2) OpenEdition}

\section{Journals}

Édition électronique

URL : https://journals.openedition.org/philosant/4203

DOI : $10.4000 /$ philosant.4203

ISSN : 2648-2789

\section{Éditeur}

Éditions Vrin

\section{Édition imprimée}

Date de publication : 15 décembre 2021

Pagination : 295-297

ISBN : 978-2-7116-3036-3

ISSN : 1634-4561

\section{Référence électronique}

Corentin Tresnie, « Nicolas D'Andrès, Socrate néoplatonicien. Une science de l'amour dans le

Commentaire de Proclus sur le Premier Alcibiade », Philosophie antique [En ligne], 21 | 2021, mis en ligne le 15 avril 2021, consulté le 06 décembre 2022. URL : http://journals.openedition.org/philosant/4203 ; DOI : https://doi.org/10.4000/philosant.4203

\section{c) (i) $(9)$}

Creative Commons - Attribution - Pas d'Utilisation Commerciale - Pas de Modification 4.0 International - CC BY-NC-ND 4.0

https://creativecommons.org/licenses/by-nc-nd/4.0/ 
tandis que l'Intellect hypostatique contemple ces Formes elles-mêmes et que, partant, la différence entre les deux se situe du côté de l'objet contemplé. De la contemplation des logoi, immanents à l'âme supérieure, il faut cependant distinguer l'état proprement « mystique » de l'identification de l'âme à l'Intellect (p. 315). Une autre lettre ( $\left.{ }^{\circ} 18\right)$

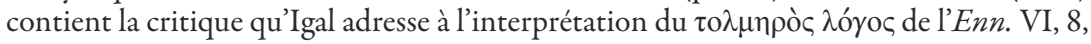
7 proposée par Armstrong.

Les lettres de Paul Henry concernent plutôt les circonstances que les questions d'établissement du texte de Plotin. On y apprend, entre autres, le rôle que le Père Henry a joué dans l'organisation des Entretiens sur les Sources de Plotin (bien qu'on se sente embarrassé en prenant connaissance des choses qui devaient demeurer « absolutely between us two », p. 75). Les lettres d'A. H. Armstrong réagissent aux questions qui intéressent leurs destinataires. C'est notamment le cas des lettres à K. Corrigan dans lesquelles Armstrong expose ses vues sur la matière et le mal dans la tradition platonicopythagoricienne. Les lettres de Jean Trouillard rendent présent le milieu des chercheurs français engagés dans l'étude du néoplatonisme, contemporains des travaux sur l'édition critique de Plotin.

Le volume rassemble de préciseuses pages biographiques et commémoratives rédigées par différents auteurs. On y trouve entre autres d'intéressantes lignes, par Martin Schwyzer, sur l'arrière-plan familial de son père ou les souvenirs, par Christopher J. R. Armstrong, des années que la famille Armstrong a passées, de 1935 à 1943, à Malte, la bataille de Malte incluse. Des plus touchants sont les passages des « Souvenirs d'un jésuite itinérant » où le Père Henry évoque le travail sur les manuscrits de Plotin et sur la tradition indirecte effectué dans sa jeunesse. « Ce fut la grâce de ma vie et me marqua indélébilement », remarque-t-il à propos de son séjour au Liban et en Palestine où il est allé pour apprendre l'arabe - « parce que j'en avais besoin pour le Plotin arabe » - et « pour vagabonder, avec ma barbe, mon keffie (châle de soie) et Agul (bande au front), circulant à pied et à dos d'âne ou de mulet » (p. 38). Heureusement, ces extraits sont publiés en français. Pour le reste, les éditeurs du volume ont pris la décision de traduire en anglais tout ce qui était écrit dans une autre langue, excepté le grec. On peut le regretter pour l'allemand de Hans-Rudolf Schwyzer et pour le français de Jean Trouillard.

Le volume contient en outre la traduction anglaise du compte-rendu de l'editio maior des Plotini Opera par Evanghelos Roussos (p. 358-364) ainsi que les lettres de celui-ci et de Paul Kalligas à H.-R. Schwyzer (à trouver entre les p. 112 et 149). Il rend également hommage à la contribution de Bertram Samuel Page dont il rapporte une lettre à E. R. Dodds et une liste des notes sur l'editio maior (p. 354-357). À travers les remarques éparses de Paul Henry, dans ses « Souvenirs » et dans ses lettres, le livre rappelle aussi l'histoire du Lexicon Plotinianum de John Herbert Sleeman et de Gilbert Pollet, plus fortunés dans la réussite de ce projet que n'étaient P. Henry lui-même en 1932 (p. 35 et 42) et E. Roussos en 1960 (p. 112 sq.).

Filip Karfík

Université de Fribourg

Nicolas D'Andrès, Socrate néoplatonicien. Une science de l'amour dans le Commentaire de Proclus sur le Premier Alcibiade, Paris, Vrin, 2020 (Textes et traditions), 304 p., ISBN : 978-2-7116-2955-8.

Les commentaires aux dialogues de Platon écrits par Proclus ne sont pas d'un usage aisé. Malgré d'excellentes éditions et traductions en français comme en anglais, souvent pourvues d'abondantes notes et commentaires, il n'est pas toujours facile de suivre le propos du Diadoque, tant le texte platonicien lui sert d'occasion au développement des 
considérations les plus diverses et parfois les plus inattendues. Celui consacré au Premier Alcibiade est de longueur et de difficulté très abordables en comparaison avec les autres commentaires, mais il est sans doute le moins unifié dans ses thématiques. L'exégète passe rapidement de la critique textuelle aux vertus purificatrices du dialogue, des bons usages érotiques à l'analyse dialectique, de l'Un aux démons, de la triade chaldaïque aux qualités pédagogiques.

L'ouvrage de N. D'Andrès, réécriture minutieuse d'une thèse de doctorat, propose de suivre de près la pensée de Proclus telle qu'elle se développe dans la première moitié de la version conservée de son commentaire, à savoir les pages 1 à 153 , ce qui correspond à peu de chose près au premier volume de l'édition Budé. Sous une apparence de monographie, il s'agit d'un véritable commentaire suivi de celui de Proclus. À partir du découpage proclien de l'Alcibiade en lemmes, il en propose une structuration plus précise.

Dans un premier chapitre, il suit la façon dont Proclus lui-même (dans le prologue au commentaire, soit In Alc. 1-15, complété par quelques passages ultérieurs) discute le plan du texte, sa place dans le cursus platonicien et ses fonctions spirituelles et cognitives, notamment en lien avec son rôle dialectique. Les deux chapitres suivants examinent l'érotique platonicienne telle que caractérisée dans le long commentaire au premier lemme (In Alc. 18-53, sur Alc. 103a1-3) à partir du Banquet et du Phèdre ainsi que de leur interprétation par Alcinoos, Plotin et Syrianus. Le quatrième chapitre se concentre sur le commentaire au second lemme (In Alc. 53-59, sur Alc. 103a3-4) et son traitement de la Providence telle qu'elle s'origine dans l'Un, est incarnée par Socrate et met en ordre les âmes particulières. Le cinquième s'intéresse au commentaire au troisième lemme (In Alc. 59-85, sur Alc. 103a4-6), qui traite de la démonologie comme lieu d'élucidation de l'érotique : discours général sur le rang des démons dans la cosmologie d'abord, fonctions purificatrices et didactiques du démon socratique ensuite. Enfin, le sixième chapitre, à propos du commentaire au quatrième lemme (In Alc. 85-93, sur Alc. 103a6-b2), ouvre aussi surl'éloge d'Alcibiade qui suit, et ses enjeux en terme d'apprentissage: rapport àl'Alcibiade historique, vertus naturelles comme expression de son potentiel, caractère approprié de l'éloge pour réfuter plus efficacement cet élève et le mener vers une érotique partagée.

Comme en témoigne l'absence de conclusion, ce dernier chapitre est une ouverture. Il étudie en effet des passages moins souvent commentés que les autres envisagés dans l'ouvrage et propose surtout des liens avec l'utilisation de la rhétorique et des logiques didactiques chez d'autres auteurs. S'il sort du propos du livre de tenter une véritable exploration de la théorie proclienne de l'enseignement, il repère déjà à ce sujet plusieurs passages clés, tant du Commentaire sur le Premier Alcibiade que d'autres textes de Proclus. Les pistes de recherche ainsi suggérées constituent une base utile pour la continuation de l'examen des questions d'enseignement chez Proclus, en l'occurrence de la façon dont il comprend et caractérise l'approche d'Alcibiade par Socrate.

Au contraire, les autres chapitres font preuve d'une abondante mobilisation de la littérature secondaire. Moins en quête d'innovation, ils réunissent avec un remarquable souci de pédagogie et de synthèse de nombreuses remarques qui constituent ensemble un état de l'art de la lecture savante de cette première partie du commentaire proclien. L'intégration des recherches réalisées entre la rédaction de la thèse et la publication est organique et fait de l'ouvrage un outil très actuel. L'érudition qui a présidé à ce travail peut s'observer dans les nombreuses notes de bas de page et dans la longue bibliographie de fin de volume.

Deux annexes précèdent celle-ci. La première est un tableau synoptique des vingt premiers lemmes de l'Alcibiade tels que découpés par Proclus, mis en parallèle avec un résumé de ce qu'a à en dire le Diadoque, ainsi que de quelques données sur la longueur 
de commentaire qu'il y consacre. La seconde annexe est une sélection de passages du Commentaire sur le Phèdre d'Hermias d'Alexandrie, qui reprennent l'enseignement présumé de Syrianus sur ce dialogue et sur l'amour en général. Ces passages sont à plusieurs reprises invoqués par l'auteur dans sa lecture de Proclus.

C'est d'ailleurs une caractéristique de l'approche textuelle choisie dans ce travail. Les arguments donnés par Proclus sont peu problématisés pour eux-mêmes, ses affirmations ne sont que ponctuellement croisées avec ce qu'on trouve ailleurs dans le Commentaire sur le Premier Alcibiade ou dans les autres textes procliens, qui ne sont toutefois pas ignorés. En revanche, les lieux parallèles chez Hermias, Olympiodore, Marinus et Damascius, ainsi que les sources ou précédents chez Platon, Plutarque, Plotin et Jamblique, sont plus systématiquement présentés, ce qui a pour effet de situer la contribution de Proclus dans sa tradition exégétique. Parfois même (par exemple dans le cas des vertus naturelles aux p. 191-193), certains concepts sont expliqués à partir de leur élaboration chez ces auteurs plutôt que par les allusions moins détaillées de Proclus. La méthode relativise donc l'originalité de ce dernier. Elle permet aussi de remarquer certains désaccords, au moins d'emphase, au sein de la tradition platonicienne, par exemple sur la question de l'amour de l'élève attendu en retour selon Hermias et Olympiodore, mais absent chez Proclus (p. 214-219).

En choisissant de ne pas aller jusqu'au commentaire ligne par ligne, ni d'aborder les questions d'établissement du texte, l'auteur gagne en clarté de présentation : les thèmes qu'il présente apparaissent ainsi avec plus de distinction qu'à la simple lecture du texte de Proclus. Cela ne l'empêche pas de consacrer une part importante de chaque section à citer les extraits les plus représentatifs du propos du Diadoque, dans la traduction de référence d'Alain-Philippe Segonds. Ce faisant, l'ouvrage peut servir d'outil de travail sur les passages auxquels il se consacre. Ce caractère est renforcé par le fait que chacune de ses propres sections est écrite de façon relativement indépendante des autres. Plus encore que l'explicitation de l'argument de Proclus, on appréciera la base documentaire fournie par chacune d'elles.

Il serait toutefois excessif de nier l'existence de tout fil rouge : comme l'indique le titre, le passage en revue des multiples sujets jusqu'ici évoqués converge vers une caractérisation de l'érotique platonicienne telle que l'envisage Proclus, et avec lui la tradition néoplatonicienne. Cette érotique est une providence par laquelle Socrate, sous l'impulsion de son démon divin, se fait l'image parfaite du Bien et tâche d'élever Alcibiade à une commune contemplation de ce Principe. À partir de ce constat, comme le montre l'auteur, on peut considérer que les dimensions rhétorique et dialectique du dialogue tel que lu par Proclus sont subordonnées, comme il le dit lui-même, à l'exigence érotique. Celle-ci permet donc de comprendre la rencontre de Socrate et Alcibiade selon les règles de poursuite amoureuse et philosophique décrites dans le Phèdre et le Banquet. Ils forment ainsi un couple paradigmatique de maître et d'élève idéaux, ce qui pourrait ouvrir la voie à une recherche plus approfondie sur leurs interactions dans les passages ultérieurs du dialogue, ainsi que sur la façon dont les interprète Proclus.

Résumons donc pour conclure le double mérite de cette étude. D’une part, il fournit à quiconque s'intéresse à la première moitié du commentaire de Proclus à l'Alcibiade ou à son intertextualité un outil à la fois complet, précis, à jour et accessible. D'autre part, il montre l'existence (parfois encore contestée) d'une authentique théorie de l'érotique pédagogique chez Proclus et dans la tradition néoplatonicienne, posant les bases d'une recherche féconde sur cette thématique jusqu'ici peu explorée. 